\title{
ARTICLE OPEN Percolative nature of the direct-current paraconductivity in cuprate superconductors
}

\author{
Petar Popčević ${ }^{1,2}$, Damjan Pelc ${ }^{3,5}$, Yang Tang ${ }^{4}$, Kristijan Velebit ${ }^{1}$, Zachary Anderson ${ }^{4}$, Vikram Nagarajan ${ }^{4}$, Guichuan Yu $^{4}$,
} Miroslav Požek (iD ${ }^{3}$, Neven Barišić ${ }^{1,3,4}$ and Martin Greven ${ }^{4}$

Despite extraordinary scientific efforts over the past three decades, the cuprate high-temperature superconductors continue to pose formidable challenges. A pivotal problem, essential for understanding both the normal and superconducting states, is to clarify the nature of the superconducting pre-pairing above the bulk transition temperature $T_{\mathrm{c}}$. Different experimental probes have given conflicting results, in part due to difficulties in discerning the superconducting response from the complex normal-state behavior. Moreover, it has proven challenging to separate common properties of the cuprates from compound-specific idiosyncrasies. Here we investigate the paraconductivity-the superconducting contribution to the direct-current (dc) conductivity - of the simple-tetragonal model cuprate material $\mathrm{HgBa}_{2} \mathrm{CuO}_{4+\delta}$. We are able to separate the superconducting and normal-state responses by taking advantage of the Fermi-liquid nature of the normal state in underdoped $\mathrm{HgBa}_{2} \mathrm{CuO}_{4+\delta}$; the robust and simple quadratic temperature-dependence of the normal-state resistivity enables us to extract the paraconductivity above the macroscopic $T_{\mathrm{c}}$ with great accuracy. We find that the paraconductivity exhibits unusual exponential temperature dependence, and that it can be quantitatively explained by a simple superconducting percolation model. Consequently, the emergence of superconductivity in this model system is dominated by the underlying intrinsic gap inhomogeneity. Motivated by these insights, we reanalyze published results for two other cuprates and find exponential behavior as well, with nearly the same characteristic temperature scale. The universal intrinsic gap inhomogeneity is not only essential for understanding the supercoducting precursor, but will also have to be taken into account in the analysis of other bulk measurements of the cuprates.

npj Quantum Materials (2018)3:42; doi:10.1038/s41535-018-0115-2

\section{INTRODUCTION}

After three decades of extensive experimental and theoretical efforts, the nature of the emergence of superconductivity in the cuprates remains controversial. ${ }^{1}$ At temperatures above the macroscopic superconducting transition temperature $T_{c}$, there exists no long-range coherence, yet traces of superconductivity remain observable, and different experimental investigations have led to widely disparate conclusions. ${ }^{2-16}$ It was recently established that the normal state of underdoped cuprates exhibits Fermiliquid charge transport ${ }^{17-21}$ and proposed that superconductivity emerges from this state in a universal percolative manner. ${ }^{8,9}$ Direct-current (dc) conductivity is a highly sensitive probe that can, in principle, provide a unique opportunity to test the consistency of these ideas. Furthermore, the effective-medium approximation required to model such a mixed regime is well established for the dc conductivity response, ${ }^{22-26}$ whereas calculations for other observables (e.g., magnetic susceptibility) are challenging. Here, we present benchmark dc conductivity data for a pristine cuprate compound, along with modeling and analysis of published results for other cuprates, that confirm the Fermi-liquid nature of the normal state and support the interpretation of percolative superconductivity emergence in a quantitative manner.
The principal problem in many previous investigations of the pre-pairing regime has been the separation of the superconducting response from the normal-state response. Different experimental probes can be sensitive to distinct aspects of the normal state. Moreover, it is well established that the underdoped cuprates also exhibit other electronic ordering tendencies, including charge-density-wave order, ${ }^{27-33}$ which has further precluded an unequivocal extraction of superconducting contributions. Prominent examples of such problems include the analysis of the Nernst effect, ${ }^{10,11}$ where a charge-order related signal might be mistaken for superconducting fluctuations, ${ }^{34,35}$ or linear magnetization and conductivity measurements, $4,12,13,36$ where the normal-state behavior is assumed to be linear in temperature, which is not necessarily the case. ${ }^{8}$ Several schemes to systematically subtract the presumed normal-state contribution have been devised, mainly based on the suppression of superconductivity with external magnetic fields. ${ }^{5,7,16}$ However, so far, only two experimental techniques are genuinely sensitive to only superconducting signals: nonlinear torque magnetization ${ }^{8}$ and nonlinear conductivity. ${ }^{9}$ Here we introduce a method to separate the dc paraconductivity from the normal-state contribution, with the resulting high-quality data readily comparable to the more involved background-free techniques.

\footnotetext{
${ }^{1}$ Institute of Solid State Physics, TU Wien, 1040 Vienna, Austria; ${ }^{2}$ Institute of Physics, Bijenička cesta 46, HR-10000 Zagreb, Croatia; ${ }^{3}$ Department of Physics, Faculty of Science, University of Zagreb, Bijenička cesta 32, HR-10000 Zagreb, Croatia and ${ }^{4}$ School of Physics and Astronomy, University of Minnesota, Minneapolis, MN 55455, USA Correspondence: Miroslav Požek (mpozek@phy.hr) or Neven Barišić (neven.barisic@tuwien.ac.at) or Martin Greven (greven@umn.edu)

${ }^{5}$ Present address: School of Physics and Astronomy, University of Minnesota, Minneapolis, MN 55455, USA

These authors contributed equally: Petar Popčević, Damjan Pelc.
}

Received: 8 December 2017 Revised: 4 August 2018 Accepted: 7 August 2018

Published online: 06 September 2018 

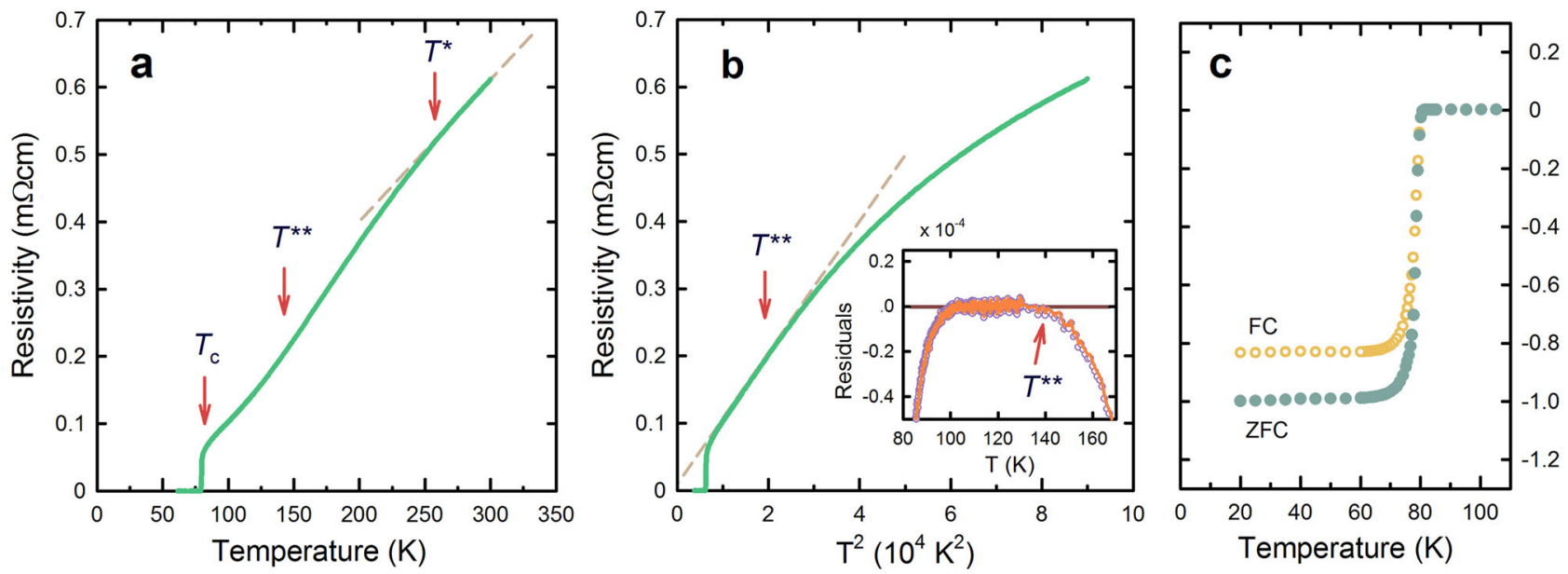

Fig. 1 a Temperature dependence of the dc resistivity of a $\mathrm{Hg} 1201$ single crystal with characteristic temperatures $T_{\mathrm{c}} \approx 80 \mathrm{~K}$ (defined here to correspond to the lowest measurable nonzero resistivity), $T^{* *} \approx 140 \mathrm{~K}$ (defined as the deviation from low-temperature quadratic behavior), and $T^{*} \approx 260 \mathrm{~K}$ (defined as the deviation from high-temperature linear behavior). $\mathbf{b}$ DC resistivity plotted versus the square of temperature and fit to $\rho=\rho_{0}+a T^{2}$ between $100 \mathrm{~K}$ and $140 \mathrm{~K}$ (dashed line), which demonstrates Fermi-liquid behavior below $T^{* *}$ and a very small residual resistivity in the zero-temperature limit (see text). The inset shows the residuals obtained upon subtracting the fit result from the data for fits between $100 \mathrm{~K}$ and $140 \mathrm{~K}$ (line) and $110 \mathrm{~K}$ and $130 \mathrm{~K}$ (symbols). The $T^{2}$ behavior prevails over a $\sim 40-\mathrm{K}$ range. c Normalized VSM magnetization measurements, obtained with an external field of $15 \mathrm{Oe}$ applied perpendicular to $\mathrm{CuO}_{2}$ planes. Solid circles: zero-field-cooled (ZFC) data; open circles: field-cooled (FC) data. These data demonstrate a well-defined superconducting transition at $T_{\mathrm{c}} \approx 80 \mathrm{~K}$ and weak vortex pinning, indicative of a low level of point disorder

A number of recent experimental investigations consistently point to a simple picture for both the normal state ${ }^{17-21}$ and the superconducting emergence regime. ${ }^{3,6,8,9}$ Measurements of transport properties, such as the dc resistivity, ${ }^{20}$ Hall angle, ${ }^{17}$ magnetoresistivity, ${ }^{19}$ and optical experiments ${ }^{21}$ unequivocally show that the mobile charge carriers behave as a Fermi liquid, even in strongly underdoped compounds. NMR investigations also find an itinerant Fermi-liquid component, along with a local, pseudogap-related signal. ${ }^{37}$ The observations that the magnetoresistivity obeys Kohler scaling with a $1 / \tau \propto T^{2}$ scattering rate ${ }^{19}$ and that the optical scattering rate exhibits conventional scaling with temperature and frequency ${ }^{21}$ are particularly clear-cut Fermiliquid signatures. Moreover, magnetization, ${ }^{8}$ high-frequency linear conductivity, $^{3,5,6,7}$ and nonlinear response measurements ${ }^{9}$ indicate that the superconducting emergence regime is limited to a rather narrow temperature range above $T_{\mathrm{c}}$ and, importantly, that it can be described with a simple percolation model. ${ }^{9}$

In the present work, we start from the fact that the normal state displays robust Fermi-liquid behavior in the pseudogap regime. We subtract its contribution to the planar resistivity with a reliability approaching the background-free techniques. With the inherent sensitivity of the dc conductivity to superconducting contributions, this enables us to obtain highly precise insight into the emergence of superconductivity and to test the percolation scenario with unprecedented accuracy. In particular, we report measurements of the dc conductivity for the cuprate $\mathrm{HgBa}_{2} \mathrm{CuO}_{4}$ $+\delta(H g 1201)$ in the underdoped part of the phase diagram. Hg1201 may be viewed as a model compound due to its simple tetragonal structural symmetry, with one $\mathrm{CuO}_{2}$ layer per formula unit, and the largest optimal $T_{\mathrm{c}}$ (nearly $100 \mathrm{~K}$ ) of all such singlelayer compounds. ${ }^{38}$ Further evidence for the model nature of $\mathrm{Hg} 1201$ comes from the observation of a tiny residual resistivity, ${ }^{20,39}$ of Shubnikov-de-Haas oscillations, ${ }^{40,41}$ and of a small density of vortex-pinning centers which has enabled the measurement of the triangular magnetic vortex lattice. ${ }^{42}$ Below the characteristic temperature $T^{* *}\left(T^{* *}<T^{*} ; T^{*}\right.$ is the pseudogap temperature), the planar resistivity of $\mathrm{Hg} 1201$ exhibits quadratic temperature dependence, $\rho \propto T^{2}$, the behavior characteristic of a Fermi liquid. $^{20}$

\section{RESULTS}

Paraconductivity measurements

We studied $\mathrm{Hg} 1201$ samples with $T_{\mathrm{c}} \approx 80 \mathrm{~K}$ (the estimated hole doping level is $p \approx 0.11$ ) that were prepared following established procedures. ${ }^{39,43}$ This particular doping level was chosen because of a relatively wide temperature range between $T^{* *}$ and $T_{c}$ in which pure quadratic-in-temperature resistivity is seen, while being reasonably far away from the doping level $(p \approx 0.09)$ where weak short-range CDW correlations are most prominent in Hg1201. ${ }^{32,33,44}$

Figure 1 shows dc resistivity data for a Hg1201 sample along with the three characteristic temperatures $T_{c}, T^{* *}$, and $T^{*}$. The purely quadratic behavior seen below $T^{* *}$ is in agreement with the Fermi-liquid character of the mobile holes. ${ }^{17-20}$ The considerable difference between $T^{* *}$ and $T_{\mathrm{c}}$ provides for a simple way to assess the superconducting paraconductivity contribution. In order to subtract the normal-state signal and obtain the purely superconducting contribution above $T_{\mathrm{c}}$, we fit $\rho(T)=\rho_{0}+a_{2} T^{2}$ to the data in a temperature range from $100 \mathrm{~K}$ to $T^{* *} \approx 140 \mathrm{~K}$, where $\rho_{0}$ is the small residual resistivity (the estimated residual resistivity ratio is $\sim 120$ ) and $a_{2}$ a constant. The resultant value of $a_{2}=9.8(1) \mathrm{n} \Omega$ $\mathrm{cm} / \mathrm{K}^{2}$ is consistent with previous measurements ${ }^{20}$ on Hg1201. A narrowing of the fit range does not change the result of our analysis, which demonstrates the robustness of the procedure (see Fig. $1 \mathrm{~b}$ inset). Furthermore, if a power law of the form $\rho(T)=\rho_{0}+$ $a T^{a}$ is fitted in the same temperature range, the exponent is $a=$ $1.98(2)$, and when the temperature range is varied from 100-140 K to $110-130 \mathrm{~K}$, it stays within $5 \%$ of this value (Supplementary Information). The fidelity of the quadratic fit is very high (Fig. 1b), which demonstrates that indeed in this temperature range, the only contribution to the resistivity is the Fermi-liquid temperature dependence. We may therefore safely extrapolate the fit to $T_{\mathrm{c}}$ to obtain the underlying normal-state contribution.

Inversion of the experimentally determined resistivity and subtraction of the extrapolated quadratic temperature dependence then gives the superconducting paraconductivity contribution, $\Delta \sigma_{\mathrm{dc}}$, shown in Fig. 2 . We present results for the same sample as above, which was chosen from a larger batch of crystals with $T_{\mathrm{c}} \approx 80 \mathrm{~K}$ due to its well-defined superconducting transitions-for $\mathrm{Hg} 1201$, the sample-contacting procedure often induces spurious 
doping of the sample surface, ${ }^{20}$ which can "short out" the current path at temperatures above the nominal $T_{\mathrm{c}}$ and artificially broaden the transition. Such samples are not considered here, although they give similar results, except in a narrow $(<1-2 \mathrm{~K})$ temperature

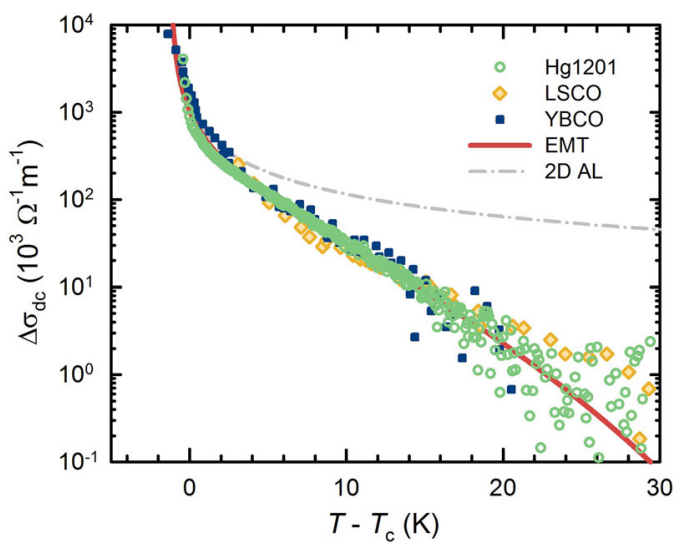

Fig. 2 DC paraconductivity for underdoped $\mathrm{Hg} 1201 \quad\left(T_{\mathrm{c}} \approx 80 \mathrm{~K}\right.$; circles), obtained by subtracting Fermi-liquid normal-state behavior from the measured resistivity, compared to the paraconductivity of underdoped YBCO ( $T_{\mathrm{c}}=47 \mathrm{~K}$; squares) and LSCO $\left(T_{\mathrm{c}}=28 \mathrm{~K}, \mathrm{Sr}\right.$ doping level $x=0.1$; diamonds) obtained by analyzing the data from ref. ${ }^{52}$ following the same procedure as for Hg1201 (see text, Supplementary Information, and Fig. S2). The paraconductivity exhibits strong exponential-like temperature dependence with a universal slope. The full line is the prediction of the superconducting percolation model obtained with effective-medium theory from a direct comparison with the Hg1201 data. The dot dashed line indicates quasi-two-dimensional Gaussian Aslamazov-Larkin (AL) fluctuation paraconductivity (see text) range above $T_{\mathrm{c}}$. We also performed a magnetic susceptibility measurement using vibrating sample magnetometry (VSM) that shows a sharp transition, with a $T_{\mathrm{c}}$ value that agrees well with the resistive $T_{c}$ (Fig. 1c). The field-cool/zero-field-cool susceptibility ratio approaches one and is among the highest observed in the cuprates, ${ }^{39}$ which demonstrates the high quality of the sample.

In order to compare our result for $\mathrm{Hg} 1201$ to other cuprates, we use the same normal-state subtraction procedure on published resistivity data for underdoped, detwinnned $\mathrm{YBa}_{2} \mathrm{Cu}_{3} \mathrm{O}_{6+\delta}(\mathrm{YBCO})$ and underdoped $\mathrm{La}_{2-x} \mathrm{Sr}_{x} \mathrm{CuO}_{4}$ (LSCO) (see Supplementary Information for details). Remarkably, as demonstrated in Fig. 2, we find the same paraconductivity behavior as for $\mathrm{Hg} 1201$. The superconducting response clearly exhibits exponential-like temperature dependence away from $T_{c}$, consistent with prior magnetization ${ }^{8}$ and nonlinear response ${ }^{9}$ results. Notably, a similar exponential paraconductivity was previously seen in YBCO at several doping levels, but with a different normal-state subtraction scheme that involves fitting to high-magnetic-field data and yields considerably lower sensitivity. ${ }^{16}$

We emphasize five crucial points: (i) the exponential dependence is qualitatively different from the underlying normal-state power-law behavior, and hence a very robust result; (ii) this behavior is observed in three distinct compounds, with the same characteristic temperature scale, which demonstrates a remarkable degree of universality; (iii) the agreement with other experiments, some of which require no background subtraction, ${ }^{8,9}$ provides additional justification for the validity of our approach to subtract the Fermi-liquid normal-state contribution; (iv) the signalto-noise ratio of the present Hg1201 data is very high, which enables us to follow the paraconductivity over more than four orders of magnitude; (v) the exponential temperature
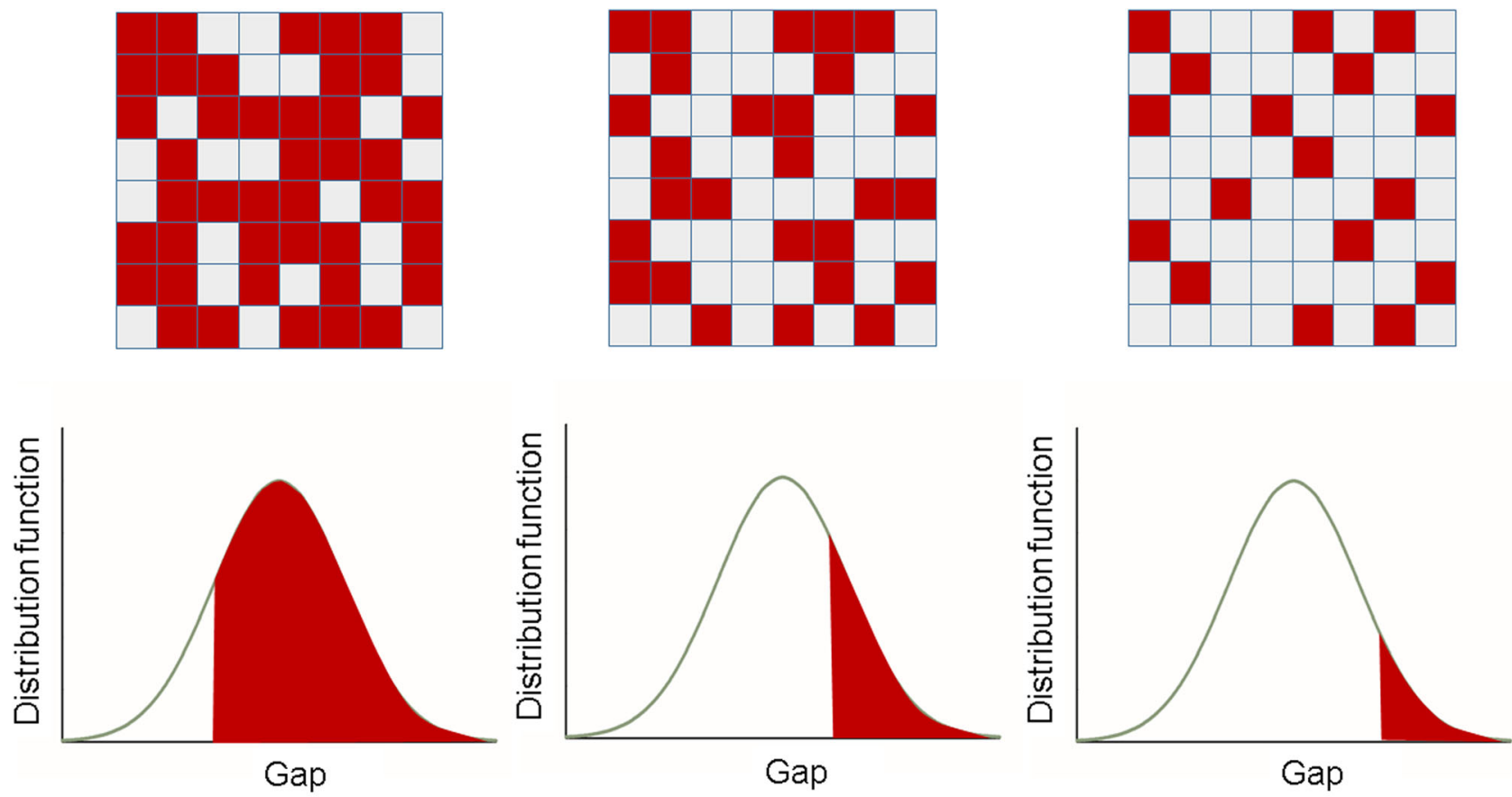

$T<T_{\mathrm{c}}$

$T \sim T_{\mathrm{c}}$

$T>T_{\mathrm{c}}$

Fig. 3 Schematic representation of the superconducting site percolation model, as a two-dimensional cross section of the full threedimensional model (upper row). Dark red patches are superconducting with vanishing resistance, whereas light gray patches have nonzero normal-state resistance. As per Eq. (1), the fraction of superconducting patches is simply obtained by integrating the local gap (i.e., $T_{c}$ ) distribution function, taken to be a Gaussian for simplicity (lower row). Note that for a typical three-dimensional percolation model the critical fraction is $\sim 0.3$ 
dependence is incompatible with standard models of superconducting fluctuations, such as Ginzburg-Landau theory. ${ }^{45}$

\section{Model}

A simple superconducting percolation model with a compoundindependent (and nearly doping-independent) underlying energy scale $k_{\mathrm{B}} \bar{\Xi}_{0}$ was recently shown to explain nonlinear response data. ${ }^{9}$ The present dc paraconductivity result provides an ideal testing ground for this model, since the model is naturally formulated in terms of the dc conductivity. In particular, the model assumes that, above $T_{c}$, the material consists of normal patches with resistance $R_{n}$, and of superconducting patches with resistance $R_{0}$ (where we will take the limit $R_{0} \rightarrow 0$ ). ${ }^{9,46}$ The fraction of superconducting patches, $P$, is temperature-dependent: at a critical fraction $P_{\pi}$ (corresponding to the critical temperature $T_{\pi}$ ), a sample-spanning superconducting cluster is formed and hence percolates. In the limit of vanishingly small currents, $T_{\pi}$ equals $T_{c}$, but in any experiment, $T_{c}$ is shifted slightly below $T_{\pi}$ due to the required nonzero currents. The temperature-dependent superconducting fraction originates from an underlying distribution of superconducting gaps, and $P$ is hence directly obtained as the temperature integral of the distribution:

$P(T)={ }_{T}^{\infty} g\left(T^{\prime}\right) \mathrm{d} T^{\prime}$

where $g\left(T^{\prime}\right)$ is the normalized local $T_{\mathrm{c}}$ distribution function. This is shown schematically in Fig. 3. For concreteness, we use the simplest (Gaussian) distribution with a full-width-at-half-maximum equal to $\Xi_{0}$, consistent with previous work. ${ }^{9}$ Other distributions, such as a skewed Gaussian, the gamma, or the logistic distributions, were also tested, but resulted in no significant differences in the outcome of the calculation-slight discrepancies between calculations with different distributions only appear in the temperature range in which the signal is close to the noise level. This insensitivity to distribution shape is simply the result of the integration over the distribution, rendering the exact shape unimportant. The dc conductivity in dependence on temperature is now obtained using effective-medium theory (EMT), ${ }^{22}$ in a form derived specifically for site percolation problems ${ }^{23}$ :

$\frac{\tilde{\sigma}_{\mathrm{dc}}-\left(1-P+P / R_{0}\right)}{1-P+P / R_{0}+\left(1 / P_{\pi}-1\right) \tilde{\sigma}_{\mathrm{dc}}} P+\frac{\tilde{\sigma}_{\mathrm{dc}}-\frac{1 / R_{0}}{P+(1-P) / R_{0}}}{P+(1-P) / R_{0}+\left(1 / P_{\pi}-1\right) \tilde{\sigma}_{\mathrm{dc}}}(1-P)=0$

where $\tilde{\sigma}_{\mathrm{dc}}$ is the conductivity normalized to the normal-state value, $R_{0}$ the resistivity of the superconducting patches (which is taken to be vanishingly small), and $P_{\pi}$ the percolation threshold. While it is known that EMT becomes unreliable in the critical regime close to the percolation threshold ${ }^{22}$ (in our case, about $2 \mathrm{~K}$ above $T_{c}$ ), we use it for simplicity in the interesting highertemperature regime away from $T_{\mathrm{c}}$. Notably, the validity of EMT has been established also in recent comparison to direct numerical calculations of the resistivity of superconductor-resistor networks in two dimensions. ${ }^{24,26}$ For networks with random (uncorrelated) distribution of superconducting links, EMT is indistinguishable from numerical calculations, while it performs slightly worse in correlated networks. We also note that the narrow critical regime, $T<T_{\mathrm{c}}+2 \mathrm{~K}$, is presumably not purely percolative, with critical exponents modified by thermal effects ${ }^{47,48}$; in order to see a discrepancy between the data and the EMT calculation, a careful power-law analysis of the critical regime would need to be undertaken, with more closely spaced measurements around $T_{\mathrm{c}}$. The investigation of criticality is thus not within the scope of the present work.

In order to obtain the limit of zero $R_{0}$, we use different small values in the numerical calculation, until no significant changes in the output are seen (typically for $R_{0}$ on the order of $10^{-5} R_{n}$ ). For simplicity, we take $R_{n}$ to be constant in the temperature interval of interest-this is appropriate, because its relative change (due to the $T^{2}$ dependence) is about $25 \%$ over a $10-\mathrm{K}$ interval, whereas the paraconductivity changes by a factor of about $10^{2}$ in the same interval. The calculated temperature dependence shown in Fig. 2 closely matches the experimental findings over the entire range of about four orders of magnitude in $\Delta \sigma$. For comparison, we also plot in Fig. 2 the standard 2D Aslamazov-Larkin (AL) paraconductivity,

$\Delta \sigma_{\mathrm{dc}}^{\mathrm{AL}}=\frac{e^{2}}{16 \hbar \mathrm{d} \epsilon}$

where $d$ is the distance between $\mathrm{CuO}_{2}$ planes (taken to be $9.5 \AA$ ) and $\epsilon=\ln T / T_{c}$. This and modified fluctuation expressions have been used in previous work ${ }^{4,16,36}$ to analyze the paraconductivity close to $T_{\mathrm{c}}$. We emphasize that this regime is not the focus of our work. In addition, as noted above, the density of our data points close to $T_{c}$ is not sufficient to perform a reliable critical exponent analysis. Yet away from $T_{\mathrm{c}}\left(T>T_{\mathrm{c}}+2 \mathrm{~K}\right)$, the measured exponential dependence is clearly captured by the EMT calculation, while the $\mathrm{AL}$ paraconductivity decays much more slowly. Physically, the reason for this dramatic difference is that in the percolation scenario, the effective spatial range of superconducting correlations is severely limited (by the mean superconducting patch size, that is in turn determined by $P$ ).

Effectively, the percolation calculation of the paraconductivity only has one free parameter: the width $\Xi_{0}$ of the $T_{\mathrm{c}}$ distribution. Other parameters that enter the calculation are constrained: $R_{\mathrm{n}}$ is simply the normal-state resistivity, $T_{\pi}$ is slightly larger than $T_{\mathrm{c}}$ (in the present calculation it was taken to be $T_{c}+1 \mathrm{~K}$, but we note that our definition of $T_{\mathrm{c}}$ as the lowest temperature with nonzero resistivity is somewhat arbitrary; different definitions, such as the midpoint of the transition measured by susceptibility, easily lead to a $1 \mathrm{~K}$ difference), and the critical concentration $P_{\pi}$ was taken to be 0.3 , consistent with the prior nonlinear conductivity analysis. ${ }^{9}$ The critical concentration $P_{\pi}$ is not arbitrary; it is determined by the details of the percolation model ${ }^{49}$ - site or bond percolation, percolation with or without farther-neighbor corrections, etc.and by the dimensionality of the percolation process. The model yields virtually the same temperature dependence for different values of $P_{\pi}$, with a corresponding change in $\Xi_{0}$ : a smaller $P_{\pi}$ implies a larger $\Xi_{0}$, and vice versa. We therefore cannot distinguish among specific percolation scenarios, such as two-dimensional versus three-dimensional percolation. Prior comparison between linear and nonlinear response indicated that a three-dimensional site percolation model with $P_{\pi} \approx 0.3$ is appropriate, ${ }^{9}$ leading us to use the same value here. Remarkably, the value $\Xi_{0}=26(1) \mathrm{K}$ that yields the best agreement with the data in Fig. 2 is in excellent agreement with nonlinear conductivity and microwave linear response for a number of cuprate compounds (Hg1201, LSCO, and YBCO) and for a range of doping levels. ${ }^{9}$ Furthermore, a similar universal scale is found in torque magnetization measurements on four different cuprate families, including $\mathrm{Hg} 1201{ }^{8}$

\section{DISCUSSION}

For simple-tetragonal $\mathrm{Hg} 1201$, the paraconductivity is a very sensitive probe of the emergence of superconductivity, and it is accurately described by the superconducting percolation scenario, with the same universal characteristic temperature scale observed for other observables in several different cuprate families. ${ }^{8,9}$ We demonstrate that the superconducting contribution can be simply obtained by assuming a Fermi-liquid normal state below the characteristic temperature $T^{* *}$. Furthermore, we show that this procedure can be readily applied to other underdoped cuprates for which $T^{* *}$ is sufficiently large compared to $T_{\mathrm{c}}$ and the underlying Fermi-liquid $T^{2}$ resistive behavior is not masked by effects due to low structural symmetry and/or point disorder (such nonuniversal effects are prevalent, e.g., in the bismuth-based cuprates or twinned $\mathrm{YBCO}^{17,19}$ ). Notably, below optimal doping, 
LSCO exhibits low-temperature resistivity upturns ${ }^{50}$ (typically revealed in the absence of superconductivity upon applying a high $c$-axis magnetic field), but the doping level of the sample analyzed here is sufficiently high for the upturn to be negligible in the temperature range of interest. For YBCO, such nonuniversal upturns generally appear at lower doping levels in the absence of intentionally introduced point disorder. ${ }^{51}$ The remarkable similarity of the paraconductivity for $\mathrm{Hg} 1201$, YBCO and LSCO constitutes a strong confirmation both for the validity of the subtraction scheme and of our main conclusions regarding a universal percolative superconductivity emergence in the cuprates.

The normal-state subtraction procedure is not possible for optimally doped compounds, where $T^{* *}$ becomes comparable to, or smaller than $T_{\mathrm{c}}$ and the resistivity no longer exhibits quadratic temperature dependence. ${ }^{20,52}$ Yet, we note that a recently proposed model for the normal state of the cuprates that considers the gradual (temperature-dependent) delocalization of charge ${ }^{53}$ explains the apparently $T$-linear resistivity and provides highly accurate predictions for the resistivity also near optimal doping. In principle, this insight could thus be used to extract the paraconductivity for compounds for which $T^{* *}$ is low.

The present work does not provide microscopic insight into the gap inhomogeneity and its origin, and in this respect the percolation model is phenomenological. It should furthermore be viewed as a zeroth-order approximation, since it treats the superconducting patches as essentially static, and does not take into account correlations and an explicit Josephson coupling between the patches. A more refined treatment should also consider thermal fluctuations and pair breaking in the patches, along the lines explored in the context of disordered superconducting films. ${ }^{54}$ Nevertheless, our basic percolation model provides a remarkable description of the conductivity and is highly consistent with experiments sensitive to real-space superconducting gap disorder, such as scanning tunneling microscopy, ${ }^{55,56}$ which have observed superconducting gap distributions with a width comparable to $k_{\mathrm{B}} \bar{\Xi}_{0}$. It is also qualitatively consistent with NMR results that demonstrate a considerable distribution of local electric field gradients, ${ }^{57,58}$ and with X-ray experiments that find percolative structures ${ }^{59,60}$ in oxygen-doped $\mathrm{La}_{2} \mathrm{CuO}_{4+\delta}$ and $\mathrm{YBCO}$.

The clear confirmation of the superconducting percolation scenario in the present work suggests that, fundamentally, both the normal-state carriers and superconducting emergence are rather conventional in the underdoped cuprates, once the underlying gap disorder is taken into account. Our result excludes the possibility of extended fluctuations usually associated with non-Fermi-liquid models. ${ }^{1,10-13}$ It also shows that it is difficult to observe the usual Ginzburg-Landau fluctuation regime in the conductivity, because inhomogeneity effects dominate-the percolation description holds down to temperatures very close to $T_{\mathrm{c}}$. Along with magnetometry as well as linear and nonlinear conductivity data, the basic percolation model naturally explains other seemingly unconventional features such as the "gap filling" seen in photoemission data, ${ }^{61}$ and thus provides a unifying understanding of superconducting pre-pairing in the cuprates. ${ }^{9}$ The dc conductivity measurements presented here have put the scenario to a stringent quantitative test, and hence constitute a crucial, independent confirmation in a model cuprate system. Furthermore, the universal gap distribution that gives rise to the observed paraconductivity is clearly intrinsic and unrelated to point disorder, since the three cuprates considered here exhibit considerably different point disorder characteristics. ${ }^{38}$ The robustness of our result therefore mandates a paradigm change in the field of cuprate superconductivity, namely that the itinerant carriers are well described by Fermi-liquid concepts, whereas the emergence of superconductivity is dominated by the gap inhomogeneity inherent to these lamellar oxides.

\section{METHODS}

Hg1201 samples were grown and characterized using established methods $, 39,43$ annealed in flowing nitrogen at $465^{\circ} \mathrm{C}$ for 1 month, and quenched to room temperature. The samples were cleaved and contacted by sputtering gold electrodes, which were connected to wires using silver paint resulting in a low contact resistance, as described in detail in previous work. ${ }^{39}$

The magnetization measurements were obtained using the VSM measurement system for the Quantum Design, Inc., Physical Property Measurement System (PPMS). Measurements were performed in the temperature range from 20 to $110 \mathrm{~K}$, enabling precise determination of the magnetization properties of the sample near the superconducting transition.

Electrical conductivity was measured using a true dc technique with current reversal, utilizing Quantum Design, Inc., PPMS. The current density was $0.02 \mathrm{~A} / \mathrm{m}^{2}$, chosen to be small enough to lead to negligible Joule heating, but large enough for a good signal-to-noise ratio for the paraconductivity. Temperature was stabilized before each datum was measured.

\section{DATA AVAILABILITY}

All data needed to evaluate the conclusions in the paper are present in the paper and/or Supplementary Information. Additional data related to this paper may be requested from the authors.

\section{ACKNOWLEDGEMENTS}

P.P. acknowledges funding by the Croatian Science Foundation Project No. IP-201606-7258. D.P. and M.P. acknowledge funding by the Croatian Science Foundation under Grant No. IP-11-2013-2729. The work at the TU Wien was supported by FWF project P27980-N36 and the European Research Council (ERC Consolidator Grant No. 725521). The work at the University of Minnesota was funded by the Department of Energy through the University of Minnesota Center for Quantum Materials under DESC-0016371.

\section{AUTHOR CONTRIBUTIONS}

P.P. and D.P. contributed equally to this work. P.P. performed the conductivity measurements. D.P. performed the model calculations. Y.T., K.V., Z.A., V.N., and G.Y. grew and characterized the samples. K.V. performed the magnetization measurements. D.P., N.B., and M.G. initiated the percolation analysis. D.P., N.B., and M.G. wrote the manuscript, with input from all authors. M.P., N.B., and M.G. supervised the project.

\section{ADDITIONAL INFORMATION}

Supplementary information accompanies the paper on the npj Quantum Materials website (https://doi.org/10.1038/s41535-018-0115-2).

Competing interests: The authors declare that they have no competing interests.

Publisher's note: Springer Nature remains neutral with regard to jurisdictional claims in published maps and institutional affiliations.

\section{REFERENCES}

1. Keimer, B., Kivelson, S. A., Norman, M. R., Uchida, S. \& Zaanen, J. From quantum matter to high-temperature superconductivity. Nature 518, 179-186 (2015).

2. Corson, R., Mallozzi, L., Orenstein, J., Eckstein, J. N. \& Božović, I. Vanishing of phase coherence in underdoped $\mathrm{Bi}_{2} \mathrm{Sr}_{2} \mathrm{CaCu}_{2} \mathrm{O}_{8+\delta}$. Nature 398, 221-223 (1999).

3. Orenstein, J., Corson, J., Oh, S. \& Eckstein, J. N. Superconducting fluctuations in $\mathrm{Bi}_{2} \mathrm{Sr}_{2} \mathrm{Ca}_{1-\mathrm{x}} \mathrm{Dy}_{\mathrm{x}} \mathrm{Cu}_{2} \mathrm{O}_{8+\delta}$ as seen by terahertz spectroscopy. Ann. Phys. 15, 596-605 (2006).

4. Silva, E., Sarti, S., Fastampa, R. \& Giura, M. Excess conductivity of overdoped $\mathrm{Bi}_{2} \mathrm{Sr}_{2} \mathrm{CaCu}_{2} \mathrm{O}_{8+\mathrm{x}}$ crystals well above $T_{\mathrm{c}}$. Phys. Rev. B 64, 144508 (2001).

5. Grbić, M. S. et al. Microwave measurements of the in-plane and c-axis conductivity in $\mathrm{HgBa}_{2} \mathrm{CuO}_{4+\delta}$ : discriminating between superconducting fluctuations and pseudogap effects. Phys. Rev. B 80, 094511 (2009).

6. Bilbro, L. S. et al. Temporal correlations of superconductivity above the transition temperature in $\mathrm{La}_{2-\mathrm{x}} \mathrm{Sr}_{\mathrm{x}} \mathrm{CuO}_{4}$ probed by terahertz spectroscopy. Nat. Phys. 7, 298-302 (2011) 
7. Grbić, M. S. et al. Temperature range of superconducting fluctuations above $T_{\mathrm{c}}$ in $\mathrm{YBa}_{2} \mathrm{Cu}_{3} \mathrm{O}_{7-\delta}$ single crystals. Phys. Rev. $B$ 83, 144508 (2011).

8. $\mathrm{Yu}, \mathrm{G}$. et al. Universal superconducting precursor in the cuprates. Preprint at https://arxiv.org/abs/1710.10957 (2017).

9. Pelc, D. et al. Emergence of superconductivity in the cuprates via a universal percolation process. Preprint at https://arxiv.org/abs/1710.10219 (2017).

10. Xu, Z. A., Ong, N. P., Wang, Y., Kakeshita, T. \& Uchida, S. Vortex-like excitations and the onset of superconducting phase fluctuation in underdoped $\mathrm{La}_{2-x} \mathrm{Sr}_{x} \mathrm{CuO}_{4}$. Nature 406, 486-488 (2000).

11. Wang, Y., Li, L. \& Ong, N. P. Nernst effect in high- $T_{\mathrm{c}}$ superconductors. Phys. Rev. $B$ 73, 024510 (2005).

12. Wang, $\mathrm{Y}$. et al. Field-enhanced diamagnetism in the pseudogap state of the cuprate $\mathrm{Bi}_{2} \mathrm{Sr}_{2} \mathrm{CaCu}_{2} \mathrm{O}_{8+\delta}$ superconductor in an intense magnetic field. Phys. Rev. Lett. 95, 247002 (2005).

13. Li, L. et al. Diamagnetism and Cooper pairing above $T_{\mathrm{c}}$ in cuprates. Phys. Rev. $B$ 81, 054510 (2010).

14. Dubroka, A. et al. Evidence of a precursor superconducting phase at temperatures as high as $180 \mathrm{~K}$ in $\mathrm{RBa}_{2} \mathrm{Cu}_{3} \mathrm{O}_{7-\delta}(\mathrm{R}=\mathrm{Y}, \mathrm{Gd}$, Eu) superconducting crystals from infrared spectroscopy. Phys. Rev. Lett. 106, 047006 (2011).

15. Uykur, E., Tanaka, K., Masui, T., Miyasaka, S. \& Tajima, S. Persistence of the superconducting condensate far above the critical temperature of $\mathrm{YBa}_{2}(\mathrm{Cu}$, $\mathrm{Zn})_{3} \mathrm{O}_{y}$ revealed by $\mathrm{c}$-axis optical conductivity measurements for several Zn concentrations and carrier doping levels. Phys. Rev. Lett. 112, 127003 (2014).

16. Rullier-Albenque, F., Alloul, H. \& Rikken, G. High-field studies of superconducting fluctuations in high- $T_{\mathrm{c}}$ cuprates: Evidence for a small gap distinct from the large pseudogap. Phys. Rev. B 84, 014522 (2011).

17. Barišić, N. et al. Evidence for a universal Fermi-liquid scattering rate throughout the phase diagram of the copper-oxide superconductors. Preprint at https://arxiv. org/abs/arxiv:1507.07885 (2015).

18. Li, Y., Tabis, W., Yu, G., Barišić, N. \& Greven, M. Hidden Fermi-liquid charge transport in the antiferromagnetic phase of the electron-doped cuprate superconductors. Phys. Rev. Lett. 117, 197001 (2016).

19. Chan, M. K. et al. In-plane magnetoresistance obeys Kohler's rule in the pseudogap phase of cuprate superconductors. Phys. Rev. Lett. 113, 177005 (2014).

20. Barišić, N. et al. Universal sheet resistance and revised phase diagram of the cuprate high-temperature superconductors. Proc. Natl Acad. Sci. USA 110, 12235-12240 (2013)

21. Mirzaei, S.-I. et al. Spectroscopic evidence for Fermi liquid-like energy and temperature dependence of the relaxation rate in the pseudogap phase of the cuprates. Proc. Natl Acad. Sci. USA 110, 5774-5778 (2013).

22. Kirkpatrick, S. Percolation and conduction. Rev. Mod. Phys. 45, 574-588 (1973).

23. Nakamura, M. Conductivity for the site-percolation problem by an improved effective-medium theory. Phys. Rev. B 29, 3691-3693 (1984).

24. Caprara, S., Grilli, M., Benfatto, L. \& Castellani, C. Effective medium theory for superconducting layers: a systematic analysis including space correlation effects. Phys. Rev. B 84, 014514 (2011).

25. Bucheli, D., Caprara, S., Castellani, C. \& Grilli, M. Metal-superconductor transition in low-dimensional superconducting clusters embedded in two-dimensional electron systems. New J. Phys. 15, 023014 (2013)

26. Caprara, S. et al. Inhomogeneous multi carrier superconductivity at $\mathrm{LaXO}_{3} / \mathrm{SrTiO}_{3}$ $(\mathrm{X}=\mathrm{Al}$ or Ti) oxide interfaces. Supercond. Sci. Technol. 28, 045004 (2015).

27. Tranquada, J. et al. Evidence for stripe correlations of spins and holes in copper oxide superconductors. Nature 375, 561-563 (1995).

28. Fauqué, B. et al. Magnetic order in the pseudogap phase of high- $T_{c}$ superconductors. Phys. Rev. Lett. 96, 197001 (2006)

29. $\mathrm{Li}, \mathrm{Y}$. et al. Magnetic order in the pseudogap phase of $\mathrm{HgBa}_{2} \mathrm{CuO}_{4+\delta}$ studied by spin-polarized neutron diffraction. Phys. Rev. B 84, 224508 (2011).

30. Chang, J. et al. Direct observation of competition between superconductivity and charge density wave order in $\mathrm{YBa}_{2} \mathrm{Cu}_{3} \mathrm{O}_{6.67}$. Nat. Phys. 8, 871-876 (2012).

31. Ghiringhelli, $\mathrm{G}$. et al. Long-range incommensurate charge fluctuations in $(\mathrm{Y}, \mathrm{Nd})$ $\mathrm{Ba}_{2} \mathrm{Cu}_{3} \mathrm{O}_{6+\mathrm{x}}$. Science 337, 821-825 (2012)

32. Tabis, W. et al. Charge order and its connection with Fermi-liquid charge transport in a pristine high- $T_{\mathrm{c}}$ cuprate. Nat. Commun. 5, 5875 (2014).

33. Tabis, W. et al. Synchrotron x-ray scattering study of charge-density-wave order in $\mathrm{HgBa}_{2} \mathrm{CuO}_{4+\delta}$. Phys. Rev. B 96, 134510 (2017).

34. Cyr-Chroiniere, $\mathrm{O}$. et al. Enhancement of the Nernst effect by stripe order in a high- $T_{\mathrm{c}}$ superconductor. Nature 458, 743-745 (2009).

35. Laliberte, F. et al. Fermi-surface reconstruction by stripe order in cuprate superconductors. Nat. Commun. 2, 432 (2011).

36. Caprara, S., Grilli, M., Leridon, B. \& Lesueur, J. Extended paraconductivity regime in underdoped cuprates. Phys. Rev. B 72, 104509 (2005).
37. Haase, J. et al. Two-component uniform spin susceptibility of superconducting $\mathrm{HgBa}_{2} \mathrm{CuO}_{4+\delta}$ single crystals measured using ${ }^{63} \mathrm{Cu}$ and ${ }^{199} \mathrm{Hg}$ nuclear magnetic resonance. Phys. Rev. B 85, 104517 (2012).

38. Eisaki, H. et al. Effect of chemical inhomogeneity in bismuth-based copper oxide superconductors. Phys. Rev. B 69, 064512 (2004).

39. Barišić, $\mathrm{N}$. et al. Demonstrating the model nature of the high-temperature superconductor $\mathrm{HgBa}_{2} \mathrm{CuO}_{4+\delta}$. Phys. Rev. B 78, 054518 (2008).

40. Chan, M. K. et al. Single reconstructed Fermi surface pocket in an underdoped single-layer cuprate superconductor. Nat. Commun. 7, 12244 (2016).

41. Barišić, N. et al. Universal quantum oscillations in the underdoped cuprate superconductors. Nat. Phys. 9, 761-764 (2013).

42. Li, Y., Egetenmeyer, N., Gavilano, J. L., Barišić, N. \& Greven, M. Magnetic vortex lattice in $\mathrm{HgBa}_{2} \mathrm{CuO}_{4+\delta}$ observed by small-angle neutron scattering. Phys. Rev. $B$ 83, 054507 (2011)

43. Zhao, X. et al. Crystal growth and characterization of the model high-temperature superconductor $\mathrm{HgBa}_{2} \mathrm{CuO}_{4+\delta}$. Adv. Mater. 18, 3243-3247 (2006).

44. Hinton, J. P. et al. The rate of quasiparticle recombination probes the onset of coherence in cuprate superconductors. Sci. Rep. 6, 23610 (2016).

45. Tinkham, M. Introduction to Superconductivity (McGraw-Hill, New York, 1996).

46. Muniz, R. A. \& Martin, l. Method for detecting superconducting stripes in hightemperature superconductors based on nonlinear resistivity measurements. Phys. Rev. Lett. 107, 127001 (2011).

47. Cardy, J. Scaling and Renormalization in Statistical Physics (Cambridge University Press, Cambridge, 1996).

48. Bergman, D. J., Aharony, A. \& Imry, Y. Percolation mechanism for long range magnetic order in disordered systems. J. Mag. Mag. Mater. 7, 217-219 (1978).

49. Stauffer, D. \& Aharony, A. Introduction to Percolation Theory (Taylor \& Francis, London, 1994).

50. Ando, Y., Boebinger, G. S., Passner, A., Kimura, T. \& Kishio, K. Logarithmic divergence of both in-plane and out-of-plane normal-state resistivities of superconducting $\mathrm{La}_{2-x} \mathrm{Sr}_{x} \mathrm{CuO}_{4}$ in the zero-temperature limit. Phys. Rev. Lett. 75, 4662-4665 (1995)

51. Rullier-Albenque, F., Alloul, H., Balakirev, F. \& Proust, C. Disorder, metal-insulator crossover and phase diagram in high- $T_{c}$ cuprates. Europhys. Lett. 81, 1-6 (2008).

52. Ando, $Y$. et al. Electronic phase diagram of high- $T_{\mathrm{c}}$ cuprate superconductors from a mapping of the in-plane resistivity curvature. Phys. Rev. Lett. 93, 267001 (2004).

53. Pelc, D. et al. Unusual behavior of cuprates explained by heterogeneous charge localization Preprint at https://arxiv.org/abs/1710.10221 (2017).

54. Char, K. \& Kapitulnik, A. Fluctuation conductivity in inhomogeneous superconductors. Z. Phys. B 72, 253-259 (1988).

55. Boyer, M. C. et al. Imaging the two gaps of the high-temperature superconductor $\mathrm{Bi}_{2} \mathrm{Sr}_{2} \mathrm{CuO}_{6+\mathrm{x}}$. Nat. Phys. 3, 802-806 (2007).

56. LV, Y.-F. et al. Mapping the electronic structure of each ingredient oxide layer of high- $T_{\mathrm{c}}$ cuprate superconductor $\mathrm{Bi}_{2} \mathrm{Sr}_{2} \mathrm{CaCu}_{2} \mathrm{O}_{8+\delta}$. Phys. Rev. Lett. 115, 237002 (2015).

57. Singer, P. W., Hunt, A. W. \& Imai, T. ${ }^{63} \mathrm{Cu}$ NQR evidence for spatial variation of hole concentration in $\mathrm{La}_{2-x} \mathrm{Sr}_{x} \mathrm{CuO}_{4}$. Phys. Rev. Lett. 88, 047602 (2002).

58. Rybicki, D. et al. Spatial inhomogeneities in single-crystal $\mathrm{HgBa}_{2} \mathrm{CuO}_{4+\delta}$ from ${ }^{63} \mathrm{Cu}$ NMR spin and quadrupole shifts. J. Supercond. Nov. Magn. 22, 179-183 (2009).

59. Poccia, N. et al. Percolative superconductivity in $\mathrm{La}_{2} \mathrm{CuO}_{4.06}$ by lattice granularity patterns with scanning micro x-ray absorption near edge structure. Appl. Phys. Lett. 104, 221903 (2014).

60. Campi, G., Ricci, A., Poccia, N. \& Bianconi, A. Imaging spatial ordering of the oxygen chains in $\mathrm{YBa}_{2} \mathrm{Cu}_{3} \mathrm{O}_{6+y}$ at the insulator-to-metal transition. J. Supercond. Nov. Magn. 27, 987-990 (2014).

61. Reber, T. J. et al. Prepairing and the "filling" gap in the cuprates from the tomographic density of states. Phys. Rev. B 87, 060506(R) (2013).

Open Access This article is licensed under a Creative Commons Attribution 4.0 International License, which permits use, sharing, adaptation, distribution and reproduction in any medium or format, as long as you give appropriate credit to the original author(s) and the source, provide a link to the Creative Commons license, and indicate if changes were made. The images or other third party material in this article are included in the article's Creative Commons license, unless indicated otherwise in a credit line to the material. If material is not included in the article's Creative Commons license and your intended use is not permitted by statutory regulation or exceeds the permitted use, you will need to obtain permission directly from the copyright holder. To view a copy of this license, visit http://creativecommons. org/licenses/by/4.0/.

(c) The Author(s) 2018 